\title{
BMJ Open Cognitive bias modification for social anxiety in adults who stutter: a feasibility study of a randomised controlled trial
}

\author{
Jan McAllister, ${ }^{1}$ Sally Gascoine ${ }^{2}$ Amy Carroll, ${ }^{3}$ Kate Humby, ${ }^{1}$ Mary Kingston, ${ }^{1}$ \\ Lee Shepstone, ${ }^{4}$ Helen Risebro, ${ }^{4}$ Bundy Mackintosh, ${ }^{5}$ \\ Tammy Davidson Thompson, ${ }^{6}$ Jo Hodgekins ${ }^{4}$
}

To cite: McAllister J,

Gascoine S, Carroll A, et al. Cognitive bias modification for social anxiety in adults who stutter: a feasibility study of a randomised controlled trial. BMJ Open 2017;7:e015601. doi:10.1136/ bmjopen-2016-015601

- Prepublication history and additional material for this paper are available online. To view these files please visit the journal (http://dx.doi.org/10. 1136/bmjopen-2016-015601).

Received 8 January 2017 Revised 22 August 2017 Accepted 6 September 2017

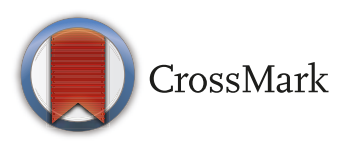

${ }^{1}$ School of Health Sciences, University of East Anglia, Research Park, Norwich, UK ${ }^{2}$ Sobell Department of Motor Neuroscience and Movement Disorders, University College London, London, UK

${ }^{3}$ Clinical Psychology, Guy's \& Thomas' NHS Foundation Trust, London, UK

${ }^{4}$ Norwich Medical School, University of East Anglia, Research Park, Norwich, UK ${ }^{5}$ Department of Psychology, University of Essex, Colchester, UK

${ }^{6}$ Speech and Language Therapy, Norwich Community Health and Care NHS Trust, Norwich, UK

Correspondence to

Dr Jan McAllister;

j.mcallister@uea.ac.uk

\section{ABSTRACT}

Objective To determine the feasibility and acceptability of a computerised treatment for social anxiety disorder for adults who stutter including identification of recruitment, retention and completion rates, large cost drivers and selection of most appropriate outcome measure(s) to inform the design of a future definitive trial.

Design Two-group parallel design (treatment vs placebo), double-blinded feasibility study. Participants: 31 adults who stutter.

Intervention Attention training via an online probe detection task in which the stimuli were images of faces displaying neutral and disgusted expressions.

Main outcome measures Psychological measures: Structured Clinical Interview Global Assessment of Functioning score; Liebowitz Social Anxiety Scale; Social Phobia and Anxiety Inventory; State-Trait Anxiety Inventory; Unhelpful Thoughts and Beliefs about Stuttering. Speech fluency: percent syllables stuttered. Economic evaluation: resource use questionnaire; EuroQol three-dimension questionnaire. Acceptability: Likert Scale questionnaire of experience of trial, acceptability of the intervention and randomisation procedure.

Results Feasibility of recruitment strategy was demonstrated. Participant feedback indicated that the intervention and definitive trial, including randomisation, would be acceptable to adults who stutter. Of the 31 participants who were randomised, 25 provided data at all three data collection points.

Conclusions The feasibility study informed components of the intervention. Modifications to the design are needed before a definitive trial can be undertaken.

Trial registration number I SRCTN55065978; Postresults.

\section{INTRODUCTION}

Stuttering is a disorder that affects the fluency of speech. The prevalence of stuttering among adults has been estimated at $0.78 \% .^{1}$ Stuttering is strongly associated with social anxiety disorder, a persistent and excessive fear of being humiliated, scrutinised or negatively evaluated in social situations, ${ }^{2}$
Strengths and limitations of this study

- The study represents the first attempt to use cognitive bias modification with adults who stutter.

- It provides valuable insights into aspects of the design and methodology that will enable us to improve these features in a future trial.

- We were unable to gather information that would explain the relatively high attrition rate among those who were randomised.

which may compromise many aspects of life including relationships, education and employment. Up to $60 \%$ of adults who are seeking treatment for stuttering experience social anxiety disorder, ${ }^{3}{ }^{4}$ compared with $6.8 \%$ of the general adult population. ${ }^{5}$ In the wider population of people who stutter, this figure may be the same, or it could be lower (eg, those with social anxiety disorder may be more likely to seek treatment because of the limitations it places on their lives) or higher (eg, avoidant tendencies associated with social anxiety disorder may make them unwilling to seek treatment). ${ }^{1}$ There is a lack of adequate provision of speech and language therapy services in the $\mathrm{UK}^{6}$ : some areas of the country have no service for adults who stutter, ${ }^{7}$ and many speech and language therapists have limited formal training in established psychological therapies. ${ }^{8}$ With increasing pressure on health service resources, an 'e-mental health' approach, ${ }^{9}$ involving online delivery of an intervention that does not require the presence of a trained therapist, could provide a cost-effective means of improving the psychological health of adults who stutter. In addition, e-mental health interventions have the potential to be more convenient for clients, including those who would otherwise have to travel long distances to access 
the service and those for whom travel is difficult, such as people with disabilities and parents with young children. However, despite the burgeoning availability of online therapies for a wide range of conditions, there is a lack of evidence regarding their effectiveness. ${ }^{10}$

The primary goal of interventions that address social anxiety in people who stutter must be improvement in mental health status. However, over half of adults who stutter cite improvements in speech fluency as their most important goal when they seek therapy. ${ }^{11}$ Some people who stutter become so ashamed of their speech disorder that they go to great lengths to disguise it via avoidance techniques; in such cases, the stutter is categorised as 'covert', 'masked' or 'interiorized'. ${ }^{12} 13$ Adults who stutter become more dysfluent in situations that increase their social anxiety, ${ }^{13}{ }^{14}$ and recent evidence suggests that the psychosocial dimension of stuttering must be addressed before speech treatment can succeed. ${ }^{15}$ Given the apparent relationship between anxiety and speech fluency, in the development of interventions for social anxiety in this group, it is desirable to include speech fluency as a secondary outcome measure.

Cognitive behavioural therapy (CBT) is an effective intervention for social anxiety disorder in adults who stutter, ${ }^{4}$ and recent trials of internet-delivered CBT for adults who stutter ${ }^{416-18}$ have produced promising results. A CBT package tailored specifically to the needs of people who stutter and delivered by a clinical psychologist improved the psychological health of participants, ${ }^{4}$ and an e-mental health version similarly resulted in improved psychological outcomes and achieved good levels of compliance. ${ }^{16-18}$ However, CBT does not work for everyone, ${ }^{19}$ and in its traditional face-to-face mode of delivery, it requires the availability of a suitably trained health professional. Furthermore, despite the negative correlation between social anxiety levels and speech fluency in adults who stutter, ${ }^{13} 14$ to the authors' knowledge, there is no evidence that using CBT to alleviate social anxiety also improves speech fluency. It has been speculated that the absence of improvements in fluency after CBT may be because once social anxiety disorder was eliminated, participants no longer feel the need to improve fluency by applying speech restructuring techniques learnt in therapy. ${ }^{4}$ Alternatively, the controlled and deliberate processing of threat that is one mechanism in $\mathrm{CBT}^{20}$ may reduce the attentional capacity required for applying speech restructuring techniques. Whatever the explanation for the absence of fluency improvements, given these limitations of CBT, is desirable to explore other forms of e-therapy that could be used instead of, or in conjunction with, CBT or other psychological interventions. ${ }^{46-18}$ An intervention approach that relied on automatic, unconscious mechanisms rather than controlled and deliberate processing might allow speech fluency to improve when social anxiety is decreased.

Evidence from cognitive research indicates that anxious and non-anxious individuals deploy attention differently when processing information. For example, in the emotional Stroop task, in which participants have to name the colour in which words are printed as quickly as possible, anxious individuals have longer colour-naming times for words with negative connotations (such as failure) than for neutral words (such as feature).$^{21}$ This finding is usually interpreted as reflecting interference between the automatic processing of the emotional content and the colour-naming process, though other explanations have been proposed. ${ }^{22}$ Attentional biases feature heavily in cognitive models of social anxiety. ${ }^{23}$ Selectively attending to threat causes and maintains feelings of anxiety. ${ }^{24}$ In particular, socially anxious people have been shown to display attentional biases towards negative facial expressions in the early stages of processing. ${ }^{25}{ }^{26} \mathrm{~A}$ technique that has been used to demonstrate this is called probe detection. For example, in one study that used probe detection, ${ }^{26}$ participants were shown pairs of faces at very brief exposure times; the two faces might both have a neutral expression, or one face might have a neutral expression and the other an emotional expression (angry or happy). The participants were asked to respond to a visual probe (an upward-pointing or downward-pointing arrow) that appeared in the location previously occupied by one of the faces. A participant who was already attending to an area of the screen where the probe appears should respond relatively quickly to the probe; therefore, relatively short reaction times would indicate a bias towards the stimulus that previously appeared at that location, and relatively long reaction times a bias away from the earlier stimulus. Non-anxious individuals had an attentional bias towards happy faces and away from threatening faces, while those with high levels of social anxiety exhibited the opposite pattern, attending towards threatening faces and away from happy faces. ${ }^{26}$ There is evidence that non-stuttering anxious people are initially hypervigilant for potential sources of threat, but after about half a second, they then reorient attention away from the threat towards more neutral stimuli. ${ }^{27} 28$ While initial hypervigilance for threat would increase the individual's awareness of and anxiety about the threat that they are concerned about, reorientation of attention away from threat stimuli prevents them from exploring and potentially reappraising feared stimuli in a way that could help them to overcome their fear.

Some findings have suggested that people who stutter also have an attentional bias towards negative stimuli during the early stages of processing. On the emotional Stroop task, adults who stutter had longer reaction times to words with negative emotional connotations than non-stuttering controls ${ }^{29}{ }^{30}$; in one study, ${ }^{29}$ adults who stutter had higher levels of both state and trait anxiety than controls, while in the other, ${ }^{30}$ no measures of anxiety were reported. In a probe detection task, children and adolescents who stutter and who met screening criteria for social anxiety disorder were biased towards looking at schematic faces with sad expressions, unlike non-anxious participants who stutter, who were biased away from such faces. ${ }^{31}$ However, in another probe detection task, 
adults who stutter exhibited the same attentional patterns as non-stuttering controls; the stuttering group did not differ from controls on a measure of social anxiety, but had significantly higher levels of trait anxiety. ${ }^{22}$ Another study found that in a more naturalistic setting where speakers had access to visual feedback from an audience when they were giving a speech, adults who stutter looked less at audience members compared with controls ${ }^{32}$; the stuttering group scored significantly higher than the controls on a measure of social anxiety. The latter finding may reflect a later stage of processing than the other tasks.

Hypervigilance for social threat can be treated using an approach called attention training, ${ }^{33} 34$ which retrains processing patterns so that attention is automatically focused away from disapproving facial expressions, with a concomitant improvement in levels of social anxiety. ${ }^{21} 35$ For example, using the probe detection paradigm, when the letter probe systematically replaced neutral rather than negative faces, thus training individuals to attend away from social threat, there was a significant likelihood that participants would no longer experience social anxiety disorder. ${ }^{34}$

Although attention training has been successfully used in non-stuttering individuals with social anxiety disorder, there have been no studies to date investigating the use of attention training to address social anxiety in adults who stutter. It is unknown how this intervention would be received by individuals who stutter and whether they would even be interested in taking part in such a study. Recruitment into intervention trials can be difficult for people experiencing mental health problems ${ }^{3637}$ and it could be the case that having both a stutter and social anxiety may make participation even more difficult. The Medical Research Council' Complex Interventions Framework (https://www.mrc.ac.uk/documents/pdf/ complex-interventions-guidance/) suggests the use of feasibility studies in order to refine the study design and to avoid problems around recruitment, retention, acceptability, compliance and other aspects of delivery in the larger study. Therefore, the present feasibility study was conducted to inform the design of a future clinical trial. Specifically, we aimed to: develop and assess recruitment strategies; assess the suitability of outcome measures for a subsequent trial; estimate SD for outcome measures to inform subsequent sample size calculations; estimate the costs and benefits associated with attention training for adults who stutter; and investigate whether both the intervention and a subsequent randomised controlled trial were likely to be acceptable to the target group, including the acceptability of random allocation.

\section{METHODS}

\section{Design}

Two-group parallel design (treatment vs placebo), double-blinded, with three data collection points (baseline, post-treatment (4 weeks), follow-up 2 (4 months)).

\section{Participants and recruitment}

As this was a feasibility study, no formal sample size calculation was carried out, but consistent with recommendations for sample sizes in feasibility studies, ${ }^{38}$ we aimed to recruit a total of 60 participants. We employed a variety of recruitment methods over a 9-month period. The study was publicised on the website and social media of the British Stammering Association www.stammering.org and via their social media, newsletters, open days and self-help groups; posters and flyers were displayed in local supermarkets, libraries, general practitioner (GP) practices, speech and language therapy clinics, pharmacies and Job Centres and at various venues within the University of East Anglia and local colleges; a press release was distributed, resulting in radio and TV interviews; we publicised the trial via the study's own website and its Twitter account, including a sponsored tweet, and study information was retweeted to over 4000000 Twitter users; we conducted a search and mail-out using National Health Service (NHS) records (GP surgeries and speech and language therapy (SLT) clinics) via searches of data sets using Read Codes 1B92, 2B49, E270 and Eu9y5, which refer to stuttering/ stammering (see https://digital.nhs.uk/): we publicised the trial in various events for local speech and language therapists. People who were interested in participating were invited to contact the research team and were then sent an information pack if they had not already received this (eg, via the search and mail-out process or by access the study website). The information pack contained a screening questionnaire which they were asked to return to the study team. The screening questionnaire included the Social Phobia Inventory (SPIN) ${ }^{39}$ a widely used screening instrument for social anxiety disorder, and also gathered demographic information and details about how respondents heard about the study, their preferred means of contact, their level of computer literacy, current involvement in stuttering or social anxiety research and comorbidity and treatments.

\section{Inclusion and exclusion criteria}

Inclusion

Participants had to be at least 18 years old and meet the criteria for risk of social anxiety disorder on the SPIN. ${ }^{39}$ An additional criterion relating to stuttering rate when measured over the telephone was removed when informal feedback suggested that it was a barrier to recruitment.

\section{Exclusion}

Potential participants were excluded if they reported having had CBT during the previous 6 months or speech treatment during the previous 12 months, had an intellectual disability, were currently at risk of self-harm or suicide, were currently using benzodiazepines or were unwilling or unable to maintain a stable dose of any extant psychotropic medication for the duration of the trial. 


\section{Intervention and placebo conditions}

Participants were randomly allocated in blocks of four or six to the two conditions using a function within the trial data management system at the Norwich Clinical Trials Unit. Apart from the research administrator, all other individuals were blinded after assignment.

\section{Intervention}

The intervention consisted of an online computer task. This was programmed and managed by Cambridge Brain Sciences (www.cambridgebrainsciences.com) via their CBSTrials.com platform, which enabled the project team to monitor compliance and issue reminders to participants if necessary, using the preferred means of contact indicated by the participant (email, phone, text or letter).

A set of 16 face images $(63 \mathrm{~mm} \times 44 \mathrm{~mm})$, representing eight individuals (four men and four women, representing a range of ages), was selected from the FACES database $^{40}$ (http://faces.mpib-berlin.mpg.de/album/ escidoc:57488). For each individual, one image displayed a disgusted expression and the other a neutral expression. The computer task involved presenting pairs of faces simultaneously.

Each 5 min computer session began with a set of practice trials (in the experimental design sense) followed by the intervention, which consisted of 160 trials. The start of a trial was indicated by the appearance of a fixation cross in the centre of the screen, which remained for $500 \mathrm{~ms}$ and on which participants were instructed to focus. A pair of faces was then presented for $500 \mathrm{~ms}$. The images within a pair represented a single individual. Each pair comprised either one face displaying a neutral expression and one displaying a disgusted expression (80\% of trials), or two faces displaying a neutral expression. The two faces were separated by 5 degrees of visual angle and were displayed centrally on the computer screen on a grey background. The faces then disappeared and a letter $\mathrm{E}$ or $\mathrm{F}$ appeared in the location previously occupied by one face. Faces, probe letters and probe positions were counterbalanced. Participants were instructed to respond to the letter probe as quickly and as accurately as possible by pressing the appropriate key on their computer keyboard. In the intervention condition, the letter only ever replaced neutral faces, never disgusted ones.

Participants were asked to complete the online computer task twice a week for 4 weeks. They were given an opportunity to practise a similar task in the baseline data collection session, at which they could ask the researcher questions if necessary. They were given the option of carrying out their eight sessions at home or at the Clinical Trials Unit of the University of East Anglia; all opted to do so at home. To ensure that the visual material was displayed in the same way regardless of the computer they were using, participants were asked to indicate the dimensions of their computer screen at the start of each session.

\section{Placebo}

The placebo condition was identical to the experimental condition, except that the letter probe was equally likely to replace either expression.

\section{Ethics and informed consent}

Ethical approval for the study was granted by the local research ethics committee (National Research Ethics Committee East of England-Cambridge South, ref: 12/ $\mathrm{EE} / 0272$ ). All ethical and research governance procedures were in place before the trial began. All participants gave individual written consent to take part in the trial.

\section{Data collection}

Data collection took place at three time points: baseline (prior to commencement of the intervention), post-treatment (approximately 1 week after the final computer session) and follow-up (after a further 3 months). Outcome measures were as follows:

\section{Psychological measures}

The following measures were collected at all three time points.

Structured Clinical Interview for DSM-IV-TR Axis I Disorders, Research Version, Non-Patient Edition (SCID). ${ }^{41}$ The full interview was administered at baseline; only the Social Anxiety section of the interview was administered at the other two time points. The Global Assessment of Functioning score is reported here.

Liebowitz Social Anxiety Scale, ${ }^{42}$ a 24-item clinician-administered assessment that focuses on performance anxiety in social situations. The assessment yields separate measures for the anxiety experienced by the participant in different situations and the extent to which they avoid those situations.

Social Phobia and Anxiety Inventory (SPAI), ${ }^{43}$ a 45 -item self-report assessment that considers specific somatic symptoms, cognitions and behaviours related to social anxiety.

State-Trait Anxiety Inventory (STAI), ${ }^{44}$ a 40 -item self-report assessment that distinguishes between the person's temporary, situation-related (state) anxiety and their relatively enduring disposition to experience (trait) anxiety.

Unhelpful Thoughts and Beliefs about Stuttering (UTBAS), ${ }^{45}$ a 66-item self-report measure that asks about unhelpful thoughts and beliefs that are often reported by people who stutter in clinic.

\section{Speech fluency}

Speech fluency was measured to enable comparison with prior research ${ }^{4}$ and to determine whether changes in social anxiety that might emerge as a result of the intervention had an impact on this variable, since prior research had suggested a correlation between social anxiety and speech fluency. ${ }^{13}$ The percent syllables stuttered speech fluency measure ${ }^{46}$ was based on an audio recording lasting approximately $2-3 \mathrm{~min}$ collected at each of the three time points. The speech sample was elicited by the interviewer following training by the fifth 
author, a speech and language therapist specialising in disorders of fluency. Participants were asked to talk about a topic of their own choosing or, if they were unable to think of a topic, the interviewer suggested several possibilities, avoiding topics that might cause emotional distress. At all three sessions, the speech sample was collected early in the interview, before the SCID. The calculation of percent syllables stuttered was carried out by the fifth author.

\section{Economic evaluation}

To inform the design of a full economic evaluation as part of a future definitive trial, we estimated questionnaire completion rates, sought to identify how and what cost and effect data would be collected and sought to identify large cost drivers, where a complete case analysis was ${ }^{47}$ performed with regard to the latter.

Costs were calculated from the perspective of the NHS and personal social services. ${ }^{48}$ Healthcare resource use data were elicited through a self-completed questionnaire administered at baseline and at 4-month follow-up. The questionnaire monitored levels of contact with different healthcare professionals (eg, speech and language therapists, counsellors and GP services), medications and hospital visits and admissions. Costs were assigned to each item of resource use using unit costs of health and social care ${ }^{49}$ and NHS Reference Costs data, ${ }^{50}$ estimated at 2012/2013 financial year levels (British pounds). The cost of the intervention itself was not included given that the intervention had already been developed as part of this study and was considered to have a negligible ongoing cost.

The EuroQol five-dimension questionnaire $\left(\right.$ EQ-5D-3L) ${ }^{51}$ including the visual analogue scale (EQ-VAS) was used to measure health-related quality of life for which responses were sought at baseline, 5 weeks and 4 months. EQ-5D-3L states were converted into utility scores using the York A1 tariff, ${ }^{52}$ where 0 is equivalent to death and 1 is full health. Within-trial quality-adjusted life year (QALY) scores were subsequently estimated using the total area under the curve approach. ${ }^{53}$

\section{Recruitment route}

To identify the optimum mode of recruitment, randomised participants were asked how they heard about the trial.

\section{Acceptability}

A self-completed structured feedback questionnaire using 5-point Likert responses was administered post-treatment to elicit participants' views and experience of participating in the trial (eg, screening, completion of outcome measures), acceptability of the intervention (completion of the computerised task) and randomisation.

After follow-up measures were completed, participants were informed of their allocation and asked whether they thought it was reasonable that they had been allocated to this condition.
Participants had the opportunity to make open-ended comments both post-treatment and at follow-up about their experience of the intervention and participation in the trial.

\section{Analysis of psychological and fluency measures}

Although this was a feasibility study, an analysis was carried out to provide an initial estimate of efficacy for each outcome. The between-group difference was expressed as a difference in means together with a 95\% CI. In addition, we used Generalised Estimating Equations (GEEs) to estimate potential intervention effects based on the two follow-up time points simultaneously. This approach incorporates the correlation between time points and does not need an assumption of independence. The models included the baseline values of the dependent variable, time point, study group and a time-by-study group interaction term. These models assumed normally distributed outcomes. These analyses were based on the 'intention-to-treat' population; a secondary analysis was carried out based on the intervention actually received. The pooled within-group SD for each outcome was calculated, together with $95 \%$ CIs, to inform the sample size of future trials.

\section{RESULTS \\ Participant recruitment and retention}

A total of 312 information packs containing screening questionnaires were sent out: 203 were sent to people identified via NHS records, 109 to people requesting packs after having heard about the study through other routes described above. Forty-nine people ( $16 \%$ of those requesting packs) completed the screening questionnaire. Figure 1 shows the Consolidated Standards of Reporting Trials (CONSORT) flowchart detailing recruitment and retention. Of those who did not meet inclusion criteria, 14 did not meet criteria for risk of social anxiety disorder, one person had received treatment for stuttering in the previous 12 months and one was unwilling or unable to maintain a stable dose of any extant psychotropic medication for the duration of the trial. Thirty-one participants were randomised to the trial, and 25 provided information at all three data collection points. Ten participants needed to be sent at least one reminder to carry out a computer session: eight participants received a single reminder, one participant received reminders about two sessions and one participant was sent reminders about seven sessions. Table 1 shows the baseline characteristics of those who were randomised. There were imbalances at baseline (eg, in age), but given the relatively small sample size, these are not unexpected.

Participants were asked how they heard about the trial. In terms of participants randomised, the British Stammering Association was the most effective recruitment route $(n=9)$, followed by posters in supermarkets $(\mathrm{n}=5)$, GP surgeries (5) or SLT clinics (2), GP search and mail-out (2) and Twitter (2). Six other methods (study 


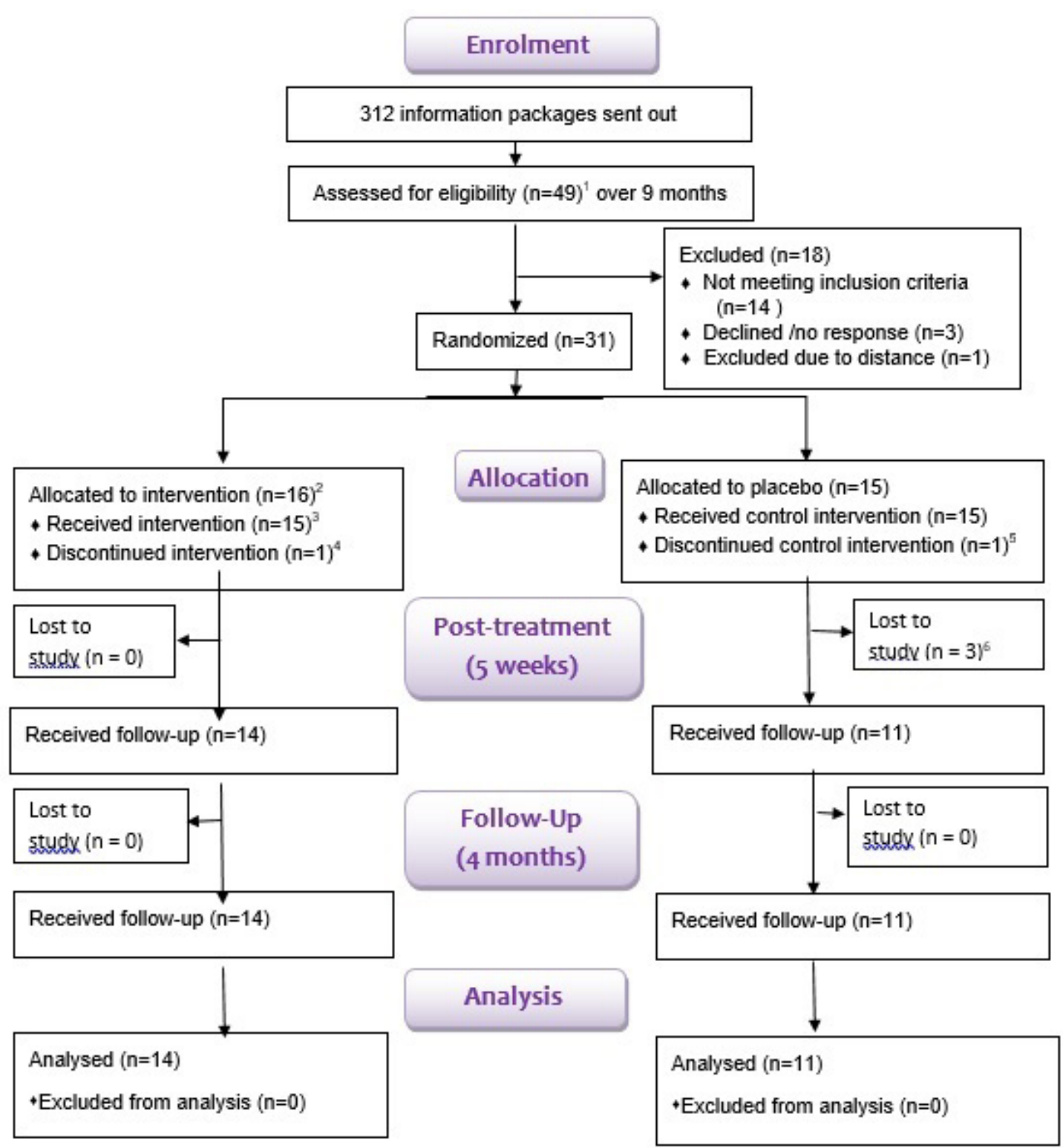

\footnotetext{
${ }^{3}$ Two further people applied shortly after randomization closed. Both would have proved eligible for inclusion

${ }^{2}$ Figure includes one person allocated to intervention group but who received placebo in error

${ }^{3}$ One person did not start intervention task

"One person started task but did not complete (reason unknown as contact lost)

${ }^{5}$ Did not complete intervention task (reason unknown as contact lost)
}

Figure 1 CONSORT flow diagram of the trial conduct.

website, local media, newsletter, SLT clinic search and mail-out, university publicity, word of mouth) resulted in a single participant each.

\section{Psychological and fluency outcome measures}

Although all participants had scores consistent with risk of social anxiety disorder according to the SPIN, only three met the full diagnostic criteria in the SCID baseline interview. The SCID excludes a diagnosis of social anxiety disorder in cases where the anxiety is attributable to stuttering, but some researchers in the field of stuttering have modified this definition to include such cases. ${ }^{54} 55$ According to this modified definition, 17 participants (seven in the attention training condition and 10 in the placebo condition) met the criteria for a diagnosis of social anxiety disorder.

Table 2 summarises data from the psychological and speech fluency outcome measures. The crude betweengroup mean differences are presented at 5-week and 4-month follow-up with 95\% CIs. These interval estimates are wide and in all but one case included 0 (ie, the differences were not statistically significant at the $5 \%$ level). In the case of the SCID, however, a statistically significant difference was seen at 4 months. The GEE models, however, adjusting for baseline scores, suggested 
Table 1 Baseline characteristics

Intervention $(n=16)$ Control $(n=15)$

\begin{tabular}{|c|c|c|}
\hline \multicolumn{3}{|l|}{ Demographics } \\
\hline \multicolumn{3}{|l|}{ Age } \\
\hline Mean (SD) & $48.3(16.2)$ & $39.7(16.0)$ \\
\hline \multicolumn{3}{|l|}{ Gender } \\
\hline $\begin{array}{l}\text { Male } \\
\text { Female }\end{array}$ & $\begin{array}{c}15(94 \%) \\
1(6 \%)\end{array}$ & $\begin{array}{l}10(67 \%) \\
5(33 \%)\end{array}$ \\
\hline \multicolumn{3}{|l|}{ Ethnicity } \\
\hline $\begin{array}{l}\text { White } \\
\text { Asian } \\
\text { Black }\end{array}$ & $\begin{array}{c}13(87 \%) \\
1(7 \%) \\
1(7 \%)\end{array}$ & $\begin{array}{l}14(93 \%) \\
1(7 \%) \\
0\end{array}$ \\
\hline \multicolumn{3}{|l|}{ Computer literacy } \\
\hline $\begin{array}{l}<5 \\
5-6 \\
>6\end{array}$ & $\begin{array}{l}3(19 \%) \\
7(44 \%) \\
6(37 \%)\end{array}$ & $\begin{array}{l}5(33 \%) \\
7(47 \%) \\
3(20 \%)\end{array}$ \\
\hline \multicolumn{3}{|l|}{ Living alone? } \\
\hline $\begin{array}{l}\text { Yes } \\
\text { No }\end{array}$ & $\begin{array}{r}2(13 \%) \\
14(87 \%)\end{array}$ & $\begin{array}{l}3(20 \%) \\
12(80 \%)\end{array}$ \\
\hline \multicolumn{3}{|c|}{ Employment status } \\
\hline $\begin{array}{l}\text { Employed } \\
\text { Self-employed } \\
\text { In education } \\
\text { Retired } \\
\text { Unemployed } \\
\text { Incapacity }\end{array}$ & $\begin{array}{l}8(50 \%) \\
2(13 \%) \\
0 \\
5(31 \%) \\
0 \\
1(6 \%)\end{array}$ & $\begin{array}{l}9(60 \%) \\
2(13 \%) \\
2(13 \%) \\
1(7 \%) \\
1(7 \%) \\
0\end{array}$ \\
\hline \multicolumn{3}{|l|}{ Recruitment route } \\
\hline $\begin{array}{l}\text { Clinical }^{*} \\
\text { Community }^{-}\end{array}$ & $\begin{array}{r}10(63 \%) \\
6(38 \%)\end{array}$ & $\begin{array}{l}9(60 \%) \\
6(40 \%)\end{array}$ \\
\hline
\end{tabular}

*British Stammering Association, GP and SLT poster, GP and SLT search and mail out.

†Supermarket poster, Twitter, study website, local media, newsletter, university publicity, word of mouth.

no difference in the SCID. There was a significant difference in the UTBAS between groups but, again, the CI is very wide, making any firm conclusions difficult to make. There were clear differences with respect to the change in scores over time between the two groups for both the SPAI and STAI, as evidenced by the significant interactions between time and study group, evidenced from the GEE model. These results are reported on an intentionto-treat basis, but it should be noted that one participant who was randomised to the treatment condition actually received the placebo. Analysing the outcomes according to intervention received made no material difference to the results.

Table 3 estimates of the pooled within-group SD (with 95\% CIs) for each of the outcomes at each follow-period to aid in sample size calculations. No agreed-upon minimally clinically important differences are available for these measures within this target population. These will be required, through consensus expert opinion, to decide an appropriate sample size for a future definitive trial.

\section{Economic evaluation}

The feasibility of conducting an economic evaluation was demonstrated by good response rates, with the Health Services Resource Use (HSRU) and EQ-5D-3L completed by $\geq 73 \%$ participants (online supplementary file 1 , table 1 ). Of the 25 participants completing the feasibility trial, 22 had complete HSRU and EQ-5D-3L questionnaire data available for complete case analysis (11 control, 11 intervention).

Given the small number of cases and the feasibility design, incremental costs and a recommendation on value for money are purposely not reported. Mean costs and QALY scores reported below should be interpreted with caution.

The source and estimated unit cost for items of resource use are displayed in online supplementary file 1, table 2. Overall, mean levels of resource use reported were low (see online supplementary file 1, table 3). Although mean number of prescriptions showed a slight increase at follow-up in both arms, these prescriptions were not deemed related to the condition. Fifteen per cent $(27 / 181)$ of total NHS healthcare contacts at baseline and 4 months concerned speech and language therapy, counselling or psychiatry and $17 \%(38 / 222)$ of prescriptions over the same period were for antidepressants (based on all available data). Total mean NHS costs per participant (not including the cost of the intervention) over the 4-month duration of follow-up were $£ 105.83$ and $£ 346.27$ for intervention and control groups, respectively (see online supplementary file 1, table 3). Costs are largely based on participants recruited from a clinical setting $(17 / 22)$, with numbers recruited from community and clinical settings evenly distributed across both arms of the trial at baseline (table 1 ). This pattern was repeated when restricted to complete cases for the economic evaluation $(\mathrm{n}=22)$ with 17 from a clinical setting (eight intervention; nine control) and 5 from a community setting (three intervention; two control).

In the Anxiety/Depression dimension of the EQ-5D-3L, $47 \%(14 / 30)$ of respondents reported any problem at baseline compared with the overall population norm of 21\%. ${ }^{56}$ Mean baseline, postintervention and follow-up scores for the EQ-5D-3L and EQ-VAS are shown in online supplementary file 1 , table 4, along with the QALY estimate. The mean EQ-5D-3L and EQ-VAS scores were similar for intervention and control arms and over all three times points. The QALY was similar in both control and intervention arms (0.283 and 0.280, respectively). It is difficult to conclude that there is any discernible pattern to these scores given the small numbers in the analyses.

\section{Acceptability}

Table 4 summarises data from the feedback questionnaire completed post-treatment, when participants had not yet been informed of their allocation. Of the 26 participants who provided data at this time point, all of those who received the placebo $(n=12)$ thought they had received 


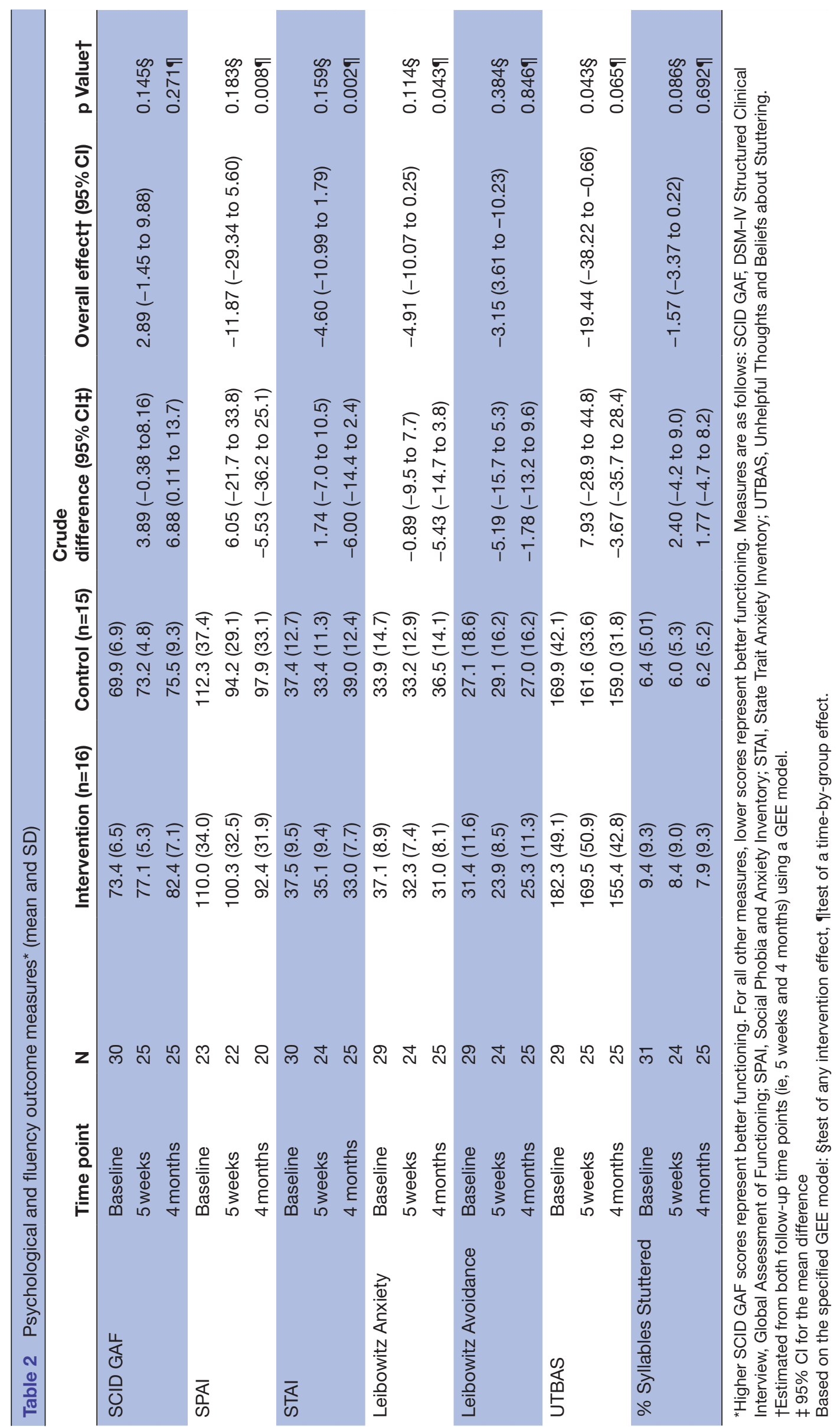


Table 3 Variability in outcome measures: pooled SD with $95 \% \mathrm{Cl}$

\begin{tabular}{lll}
\hline & 5 weeks follow-up & 4 months follow-up \\
\hline SCID & $5.13(4.00$ to 7.19$)$ & $8.12(6.31$ to 11.40$)$ \\
SPAI & $31.0(23.8$ to 44.8$)$ & $32.5(24.5$ to 48.0$)$ \\
STAI & $10.2(7.9$ to 14.5$)$ & $10.0(7.8$ to 14.1$)$ \\
Leibowitz Anxiety & $10.0(7.7$ to 14.2$)$ & $11.1(8.6$ to 15.6$)$ \\
Leibowitz Avoidance & $12.2(9.5$ to 17.3$)$ & $13.7(10.6$ to 19.2$)$ \\
UTBAS & $44.2(34.4$ to 62.0$)$ & $38.4(30.0$ to 53.9$)$ \\
\% Syllables Stuttered & $7.71(5.96$ to 10.92$)$ & $7.77(6.04$ to 10.89$)$ \\
\hline
\end{tabular}

the placebo; $11(78.6 \%)$ of those who received the intervention also believed they had received the placebo; only 3 participants who received the intervention correctly believed that they had done so. At follow-up, they were informed of their allocation to attention training or placebo and were asked whether they thought it was reasonable that they had been allocated to this condition: only one participant (who had received attention training) felt that randomisation was unreasonable.

Participants had an opportunity to make open-ended comments both post-treatment and at follow-up. Participants made positive comments about the organisation and structure of the study, their interaction with the staff, their own perception of the effect of the treatment, the simplicity of the task, the potential value of such an intervention if effective and their enjoyment of participation. Several expressed a wish to know more about the mechanism underpinning the treatment (they received information about this after data collection was completed). One person noted that the print size of one of the questionnaires was very small. Several remarked that they understood the reasons for randomisation.

\section{Suitability of outcome measures}

Outcome measures were generally found to be suitable, but some deserve further comment. The face-to-face nature of the SCID interview caused logistical challenges in terms of availability of interviewer and participant and

Table 4 Data from participant feedback questionnaire post-treatment

\begin{tabular}{|c|c|c|c|c|c|c|}
\hline & & $\begin{array}{l}\text { Strongly } \\
\text { disagree }\end{array}$ & $\begin{array}{l}\text { Mostly } \\
\text { disagree }\end{array}$ & $\begin{array}{l}\text { Neither agree } \\
\text { nor disagree }\end{array}$ & Mostly agree & Strongly agree \\
\hline $\begin{array}{l}\text { I disliked the initial screening } \\
\text { questionnaire that I filled in. }\end{array}$ & AT & 11 & & 1 & & \\
\hline \multirow{2}{*}{$\begin{array}{l}\text { I didn't mind having my } \\
\text { speech recorded over the } \\
\text { telephone*. }\end{array}$} & AT & & 1 & 1 & 3 & 3 \\
\hline & Placebo & 1 & 1 & 2 & 4 & 3 \\
\hline $\begin{array}{l}\text { Over the time that I was } \\
\text { involved, I had to spend too } \\
\text { long having assessments } \\
\text { done at University of East } \\
\text { Anglia }\end{array}$ & Placebo & 10 & 2 & 1 & & \\
\hline $\begin{array}{l}\text { I disliked having my speech } \\
\text { recorded each time I visited } \\
\text { UEA }\end{array}$ & AT & 7 & 2 & 1 & 1 & 1 \\
\hline \multirow{2}{*}{$\begin{array}{l}\text { The computer task took up } \\
\text { too much time. }\end{array}$} & AT & 10 & 2 & & & \\
\hline & Placebo & 8 & 5 & & & \\
\hline \multirow{2}{*}{$\begin{array}{l}\text { I was happy to be randomly } \\
\text { allocated to a version of } \\
\text { computer session even } \\
\text { though I understand that it } \\
\text { might have been the placebo } \\
\text { version. } \dagger\end{array}$} & AT & 1 & & 1 & & 10 \\
\hline & Placebo & & & & 4 & 9 \\
\hline
\end{tabular}

*The telephone screen was subsequently omitted.

tAt this stage the participant had not been informed of their allocation. 
may also have had a negative impact on recruitment; in future, an equivalent online measure might be preferable. The format of the paper version of the SPAI was such that it was very easy to miss items and, as a result, there were $1.6 \%$ missing values for this measure; in the future, it would be better to use a different format or to have the researcher check the form while the participant is present. Finally, although participants generally reported that they did not object to having their speech recorded, this measure could be a barrier to recruitment to a future trial, and consideration should be given to using a self-report measure instead.

\section{DISCUSSION}

Despite a diverse recruitment strategy implemented over a period of 9 months, we were only able to randomise approximately half of our original target. Furthermore, only around $16 \%$ of the screening questionnaires that were sent out were returned. It is unlikely that our recruitment difficulties were due to a low prevalence of social anxiety disorder among adults who stutter: studies with clinical samples indicate a high prevalence, ${ }^{34}$ and studies using community samples report poor psychological health among adults who stutter. ${ }^{57}{ }^{58}$ In general, with regard to recruitment and retention, the main lesson from this study is that a future trial is likely to need an extended recruitment period and wide geographical reach, and would require a similarly diverse recruitment strategy which should involve user organisations such as the British Stammering Association. By contrast, NHS search and mail-out may be a less time-effective and cost-effective method with this client group. The difficulties experienced with recruitment are similar to those outlined in other studies recruiting individuals with mental health difficulties. ${ }^{36}{ }^{37}$ Mental health studies which have had success in recruiting participants have highlighted the importance of a flexible and individualised approach to recruitment. ${ }^{59}$

We do not know the reasons for the $20 \%$ attrition rate among those who were randomised. It is possible that they lost interest in the project because they were dubious about the likelihood of success: several participants remarked that it was difficult to see how a computer task could help with anxiety, and the majority of participants thought they had been allocated to the placebo regardless of their actual allocation. This possible barrier to recruitment would need to be addressed in any future trial, though in a manner that would not be likely to impact on effectiveness. Other factors such as the age of participants could also be explored.

With some provisos, the outcome measures were suitable and their use in a future study is feasible for the most part. The small print size of the SPAI may have led to some missing data and was commented on by one of the participants, so in a future trial, an alternative format may need to be adopted. Considering that the fears of those with social anxiety disorder centre on social interaction, perhaps it is inevitable that recruitment and retention will be challenging in a study that requires repeated face-toface interaction with members of the research team. A possible solution could be to minimise the face-to-face element by using online self-completion measures as much as possible. Consideration also needs to be given to whether and how data about speech fluency should be collected, given that dislike of voice recording could be a reason for problems of recruitment and retention. On the other hand, there could be some value in collecting speech fluency data more regularly, for example, immediately after each computer session. If a speech fluency measure is included in a future study, the duration of the speech sample as well as the mode of collection would need to be considered carefully; the short sample collected in the present study was very short and therefore might not be representative of the individual's speech, but increasing the length of the speech sample would increase participant burden and might have a negative impact on recruitment and retention. Furthermore, levels of social anxiety could be particularly high among people with a 'covert', 'masked' or 'interiorized' stutter ${ }^{12} 13$ which they keep concealed by adopting avoidance strategies, but a speech fluency measure could be misleading in their case.

We have shown that it is feasible to collect resource use and EQ-5D data from this population group. However, the mean overall costs do not suggest that this population places a particular burden on the NHS; indeed, direct costs are lower than those reported elsewhere for computerised CBT for anxiety and depression in primary care. ${ }^{60}$ It is also likely that many of the services reported would have been due to health issues not related to stuttering. Similarly, the mean EQ-5D-3L population norm score has been estimated to be $0.86,{ }^{61}$ which is again not noticeably different to the scores for the population in this study.

Feedback from participants was generally good. They reported that their interaction with the project team was positive. They found the computer task undemanding. Almost all the participants indicated that they did not view the prospect of randomisation negatively. It is likely that this form of intervention would be acceptable to potential end-users.

\section{CONCLUSIONS}

Participants in this feasibility study trial felt that the intervention would be valuable and reported few concerns about the trial itself. If a definitive Randomised Controlled Trial is developed, however, careful consideration will need to be given to participant recruitment, mode of data collection (online vs face to face) and inclusion of a speech fluency measure.

Acknowledgements The authors would like to thank the participants, the British Stammering Association and the South Norfolk Clinical Commissioning Group. 
Contributors JMcA was the $\mathrm{Cl}$; she led on the design of the study and composed the first draft of the paper. SG, AC,KH and MK had the main responsibilities associated with recruitment and data collection. LS, HR, BM, TDT and JH, as well as the authors mentioned earlier, contributed to the design and/or analysis of the study. All authors read and commented on the manuscript and approved the final draft.

Funding This paper presents independent research funded by the National Institute for Health Research (NIHR) under its Research for Patient Benefit (RfPB) Programme (Grant Reference Number PB-PG-0610-22225). The views expressed are those of the author(s) and not necessarily those of the NHS, the NIHR or the Department of Health.

Competing interests None declared.

Ethics approval NRES Committee East of England-Cambridge South.

Provenance and peer review Not commissioned; externally peer reviewed.

Data sharing statement Data from a cognitive bias experiment and a user questionnaire were also gathered. All data are available only to the study team.

Open Access This is an Open Access article distributed in accordance with the Creative Commons Attribution Non Commercial (CC BY-NC 4.0) license, which permits others to distribute, remix, adapt, build upon this work non-commercially, and license their derivative works on different terms, provided the original work is properly cited and the use is non-commercial. See: http://creativecommons.org/ licenses/by-nc/4.0/

(C) Article author(s) (or their employer(s) unless otherwise stated in the text of the article) 2017. All rights reserved. No commercial use is permitted unless otherwise expressly granted.

\section{REFERENCES}

1. Craig A, Hancock K, Tran Y, et al. Epidemiology of stuttering in the community across the entire life span. J Speech Lang Hear Res 2002;45:1097-105.

2. Association AP. Diagnostic and statistical manual of mental disorders (DSM-5): American Psychiatric Pub, 2013.

3. Iverach L, O'Brian S, Jones $\mathrm{M}$, et al. Prevalence of anxiety disorders among adults seeking speech therapy for stuttering. J Anxiety Disord 2009;23:928-34.

4. Menzies RG, O'Brian S, Onslow M, et al. An experimental clinical trial of a cognitive-behavior therapy package for chronic stuttering. J Speech Lang Hear Res 2008;51:1451-64.

5. Kessler RC, Chiu WT, Demler O, et al. Prevalence, severity, and comorbidity of 12-month DSM-IV disorders in the National Comorbidity Survey Replication. Arch Gen Psychiatry 2005;62:617-27.

6. Bercow J. The Bercow Report: A review of services for children and young people $(0-19)$ with speech, language and communication needs. 2008.

7. Association BS. Adult therapy and courses: Finding a qualified speech and language therapist. https://www.stammering.org/ help-information/topics/therapy-treatment/adults-finding-qualifiedspeech-and-language-therapis.

8. Davidson Thompson T. A Survey of Practice of Speech and Language Therapists who Work with People who Stutter: University of East Anglia, 2010.

9. Federation N. e-mental health: what's all the fuss about? http://www nhsconfed.org/resources/2013/01/e-mental-health-whats-all-thefuss-about.

10. Loucas CE, Fairburn CG, Whittington C, et al. E-therapy in the treatment and prevention of eating disorders: A systematic review and meta-analysis. Behav Res Ther 2014;63:122-31.

11. Venkatagiri HS. What Do People Who Stutter Want-Fluency or Freedom? Journal of Speech Language and Hearing Research 2009;52:500-15.

12. Cheasman C, Everard R, Simpson S. Stammering therapy from the Inside. New perspectives on working with young people and adults. 2013.

13. Onslow M. Stuttering and its treatment: Eleven lectures. 2016 https:// sydney.edu.au/health-sciences/asrc/docs/eleven_lectures.pdf.

14. Packman A. Theory and therapy in stuttering: a complex relationship. J Fluency Disord 2012;37:225-33.

15. Craig AR, Hancock K. Self-Reported Factors Related to Relapse following Treatment for Stuttering. Australian Journal of Human Communication Disorders 1995;23:48-60.
16. Helgadóttir FD, Menzies RG, Onslow M, et al. Online CBT II: A Phase I Trial of a Standalone, Online CBT Treatment Program for Social Anxiety in Stuttering. Behaviour Change 2009;26:254-70.

17. Helgadóttir FD, Menzies RG, Onslow M, et al. A standalone Internet cognitive behavior therapy treatment for social anxiety in adults who stutter: CBTpsych. J Fluency Disord 2014;41:47-54.

18. Menzies R, O'Brian S, Lowe R, et al. International Phase II clinical trial of CBTPsych: A standalone Internet social anxiety treatment for adults who stutter. J Fluency Disord 2016;48:35-43.

19. Scott J, Beck A. et alCognitive behavioural therapy. In: Kendler K McGuffin P, Wessely S, Castle D, . eds. Essential Psychiatry. 4th Edn. Cambridge: Cambridge University Press.

20. Teachman BA, Beadel JR, Steinman SA. Mechanisms of change in CBT treatment: The Wiley Handbook of Anxiety Disorders, 2014:824-39.

21. Mathews A, MacLeod C. Selective processing of threat cues in anxiety states. Behav Res Ther 1985;23:563-9.

22. Lowe R, Menzies R, Packman A, et al. Assessing attentional biases with stuttering. Int J Lang Commun Disord 2016;51:84-94.

23. Clark DM, Wells A. A cognitive model of social phobia. Social phobia: diagnosis, assessment, and treatment 1995;41:00022-3.

24. Van Bockstaele $B$, Verschuere $B$, Tibboel $\mathrm{H}$, et al. A review of current evidence for the causal impact of attentional bias on fear and anxiety. Psychol Bull 2014;140:682-721.

25. MacLeod C, Rutherford E, Campbell L, et al. Selective attention and emotional vulnerability: assessing the causal basis of their association through the experimental manipulation of attentional bias. J Abnorm Psychol 2002;111:107-23.

26. Pishyar R, Harris LM, Menzies RG. Attentional bias for words and faces in social anxiety. Anxiety, Stress \& Coping 2004;17:23-36.

27. Vassilopoulos SP. Social anxiety and the vigilance-avoidance pattern of attentional processing. Behav Cogn Psychother 1999;33:13-24.

28. Booth RW. Uncontrolled avoidance of threat: Vigilanceavoidance, executive control, inhibition and shifting. Cogn Emot 2014;28:1465-73.

29. Hennessey NW, Dourado E, Beilby JM. Anxiety and speaking in people who stutter: an investigation using the emotional Stroop task. J Fluency Disord 2014;40:44-57.

30. Lieshout $P$, Ben-David B, Lipski M, et al. The impact of threat and cognitive stress on speech motor control in people who stutter. J Fluency Disord 2014;40:93-109.

31. McAllister J, Kelman E, Millard S. Anxiety and cognitive bias in children and young people who stutter. Procedia Soc Behav Sci 2015;193:183-91.

32. Lowe R, Guastella AJ, Chen NT, et al. Avoidance of eye gaze by adults who stutter. J Fluency Disord 2012;37:263-74.

33. Amir N, Beard C, Taylor CT, et al. Attention training in individuals with generalized social phobia: A randomized controlled trial. J Consult Clin Psychol 2009;77:961-73.

34. Schmidt NB, Richey JA, Buckner JD, et al. Attention training for generalized social anxiety disorder. J Abnorm Psychol 2009;118:5-14

35. Staugaard SR. Threatening faces and social anxiety: a literature review. Clin Psychol Rev 2010;30:669-90.

36. Hughes-Morley A, Young B, Waheed W, et al. Factors affecting recruitment into depression trials: Systematic review, meta-synthesis and conceptual framework. J Affect Disord 2015;172:274-90.

37. Patterson S, Kramo K, Soteriou T, et al. The great divide: a qualitative investigation of factors influencing researcher access to potential randomised controlled trial participants in mental health settings. J Ment Health 2010;19:532-41.

38. Lancaster GA, Dodd S, Williamson PR. Design and analysis of pilot studies: recommendations for good practice. J Eval Clin Pract 2004;10:307-12.

39. Connor KM, Davidson JR, Churchill LE, et al. Psychometric properties of the social phobia inventory (SPIN). The British Journal of Psychiatry 2000;176:379-86

40. Ebner NC, Riediger M, Lindenberger U. FACES--a database of facial expressions in young, middle-aged, and older women and men: development and validation. Behav Res Methods 2010;42:351-62.

41. First MB, Spitzer RL, Gibbon M, et al; Structured Clinical Interview for DSM-IV-TR Axis I Disorders, Research Version, Patient Edition: $S C I D-I / P)$ New York: Biometrics Research, New York State Psychiatric Institute, 2002.

42. Liebowitz M, phobia S. Mod Problem Pharmacopsychiatry. 1987. 22 141173. Go to original source.

43. Turner SM, Beidel DC, Dancu CV, et al. An empirically derived inventory to measure social fears and anxiety: The Social Phobia and Anxiety Inventory. Psychol Assess 1989;1:35-40.

44. Spielberger CD, Gorsuch RL, Lushene RE, et al. State-trait anxiety inventory (STAI). BiB 1970;2010:180. 
45. St Clare T, Menzies RG, Onslow M, et al. Unhelpful thoughts and beliefs linked to social anxiety in stuttering: development of a measure. Int J Lang Commun Disord 2009;44:338-51.

46. O'Brian S, Packman A, Onslow M, et al. Measurement of stuttering in adults: comparison of stuttering-rate and severity-scaling methods. \$('.RefSINo'). each(function0\{\$(this). html(\$(this).html(+'.')\})J Speech Lang Hear Res 2004;47:1081-7.

47. Dolan P, Roberts J. Modelling valuations for Eq-5d health states: an alternative model using differences in valuations. Med Care 2002;40:442-6.

48. National Institute of Health and Clinical Excellence. Guide to the methods of technology appraisal 2013. Process and methods: NICE publications, 2013.

49. Curtis L. Unit costs of health and social care 2013: Personal Social Services Research Unit: The University of Kent, 2013.

50. Department of Health. Reference Costs 2012-2013. Department of Health: Department of Health, 2013.

51. Brooks R, Rabin R, de Charro F, et al. The measurement and valuation of health status using EQ-5D: a European perspective: Kluwer Academic Publishers, 2003.

52. Dolan P. Modeling valuations for EuroQol health states. Med Care 1997;35:1095-108.

53. Manca A, Hawkins N, Sculpher MJ. Estimating mean QALYs in trialbased cost-effectiveness analysis: the importance of controlling for baseline utility. Health Econ 2005;14:487-96.
54. Iverach L, Rapee RM. Social anxiety disorder and stuttering: current status and future directions. J Fluency Disord 2014;40:69-82.

55. Stein MB, Baird A, Walker JR. Social phobia in adults with stuttering. Am J Psychiatry 1996;153:278.

56. Kind P, Dolan P, Gudex C, et al. Variations in population health status: results from a United Kingdom national questionnaire survey. BMJ 1998;316:736-41.

57. McAllister J, Collier J, Shepstone L. The impact of adolescent stuttering and other speech problems on psychological well-being in adulthood: evidence from a birth cohort study. Int J Lang Commun Disord 2013;48:458-68.

58. Blumgart E, Tran Y, Craig A. Social anxiety disorder in adults who stutter. Depress Anxiety 2010;27:687-92.

59. Notley C, Christopher R, Hodgekins J, et al. Participant views on involvement in a trial of social recovery cognitive-behavioural therapy. Br J Psychiatry 2015;206:122-7.

60. McCrone P, Knapp M, Proudfoot J, et al. Cost-effectiveness of computerised cognitive-behavioural therapy for anxiety and depression in primary care: randomised controlled trial. $\mathrm{Br} J$ Psychiatry 2004;185:55-62.

61. Kind P, Hardman G, Macran S. UK population norms for EQ-5D: centre for health economics discussion paper. 172, 1999. 